\title{
A case of bipolar disorder developing into atypical parkinsonism and presenting with frontotemporal asymmetrical brain degeneration. A TREDEM Registry Case Report
}

\author{
Maria E. Di Battista ${ }^{1}$ (D) | Carola Dell'Acqua ${ }^{1,2}$ | Daniela Galimberti ${ }^{3,4}$ \\ Maria Serpente $^{3,4}$ | Franco Boccaletto ${ }^{5}$ | Maurizio Gallucci ${ }^{1}$ (D) \\ ${ }^{1}$ Cognitive Impairment Center, Local Health Authority n. 2 Marca Trevigiana, Treviso, Italy \\ ${ }^{2}$ Department of Neuroscience \& Padova Neuroscience Center, University of Padova, Padova, Italy \\ ${ }^{3}$ Dino Ferrari Center, University of Milan, Milan, Italy \\ ${ }^{4}$ Fondazione IRCCS Ca' Granda, Neurodegenerative Disease Unit, Milan, Italy \\ ${ }^{5}$ Nuclear Medicine Unit, Local Health Authority n. 2 Marca Trevigiana, Treviso, Italy
}

Correspondence: Maria E. Di Battista, Cognitive Impairment Center, Local Health Authority n. 2 Marca Trevigiana, Treviso, Italy.

Email: mariaelena.dibattista@aulss2.veneto.it

Keywords: atypical parkinsonism, bipolar disorder, frontotemporal lobar degeneration, progressive supranuclear palsy

\section{1 | CASE PRESENTATION}

A 67-year-old man with a diagnosis of bipolar disorder (BD) Type I since he was 35 years old, was admitted to our Memory Clinic in 2016 because, throughout the last year, his wife had noticed the onset of attentional deficits and a significant worsening of motor slowness. The patient is married and a father of two, he has worked as a naval carpenter and he was retired at the time of the first admission at our clinic. He has never smoked nor drunk alcohol. No family history for psychiatric or neurodegenerative disease was reported. The patient has been monitored by the local psychiatric services throughout his life and the psychiatric reports describe his periods of mania, lasting for 2-3 years, with the presence of: dangerous driving, sleep disorder during which the patient carried out domestic activities in the night, expansive behaviour towards women, periods of excessive prodigality for hobbies and holidays. These hypo-maniac periods were characterized by an increase of energy, impulsiveness and risky behaviour. His condition included periods of remission and periods of hypomania with extremely depressed mood during which made it hard for him to go to work. Long-acting injectable antipsychotics (Haloperidol) were initially dispensed by the psychiatric service. Subsequently, about 25 years ago, the patient was started on lithium, which was partially affective to control behavioural symptoms that remained stable in frequency for at least further 20 years. About ten years ago the patient was started with olanzapine. In the last four years, while the psychiatric complaints decreased in severity, the patient has started to develop motor slowness.

Four years ago the patient was visited by the local neurology department for signs of bradykinesia, the neurological examination performed in 2014 showed signs of akinetic-rigid parkinsonism that were believed secondary to the treatment with lithium $300 \mathrm{mg}$ twice daily and olanzapine $5 \mathrm{mg}$. The patient was well-functioning and in a condition of good psychopathological balance.

In 2016, the patient was admitted to our Memory Clinic. Neurological examination at first admission showed severe parkinsonism with moderate-to-severe limb rigidity, marked impairment of finger and toe tapping with predominance on the right side. Axial symptoms were also present and included reduced postural reflexes, marked neck rigidity and limitation of vertical eye pursuit. Dystonia or phantom limb phenomenon was absent. Therapy at admission was: Carbolithium $300 \mathrm{mg}$ 2/daily; Lorazepam $2.5 \mathrm{mg}$ /daily and Olanzapine $5 \mathrm{mg}$ /daily. Serum lithium was always within therapeutic range. 
vertical smooth limitation of eye movements turned into vertical gaze palsy. The cognitive status remained stable, with prominent executive dysfunctions. Also, the patient did not develop language or severe behavioural deficit during the observational period. The patient suddenly died at home from cardiac arrest in January 2019.

The patient's neuropsychological profile was characterized by mild impairment of attention and phonemic fluency with normal semantic fluency and memory functioning. The executive functions were severely affected. Neuropsychiatric examination revealed the presence of mild depression and apathy but no psychotic symptom, hallucination or disinhibition.

Magnetic resonance imaging revealed an asymmetric cortical atrophy with severe involvement of the left frontal and temporal regions (left insula, left lateral temporal region, left frontal lobe, especially on the dorsolateral prefrontal cortex), a moderate atrophy in the right frontal regions and in the mesial bilateral frontal regions. Also, an area of focal atrophy can be appreciated in the right posterior medial region of the parietal lobe (Figure $1 \mathrm{~A}-\mathrm{H}$ ).

Metabolic evaluation with 18-FDG-PET evidenced a diffused decrease in cortical metabolism, prominent on the left side. Areas of cortical hypometabolism include the frontal dorsolateral, parietal and left temporal lobe in toto (Figure 1I-L).

On the DatSCAN evaluation, we observed moderate and asymmetric reduction of dopamine uptake (Figure $1 \mathrm{M}$ ).

\section{Key message}

Following a long-standing history of bipolar disorder (BD), a patient presented with parkinsonism. Left frontotemporal atrophy at magnetic resonance imaging and hypometabolism at positron emission tomography with tracer 18-fluorine fluorodeoxyglucose were detected. Diagnostic criteria for Frontotemporal Dementia, progressive supranuclear palsy-parkinsonism, idiopathic Parkinson's disease or drug-induced Parkinson were doubtful. We suggest that it was a case of atypical parkinsonism due to frontal degeneration, after a long-standing history of BD.

Progranulin plasma levels were found to be within the normal range, thus, no further GRN sequencing was carried out; no expansion of C9ORF72 hexanucleotide repeats was detected.

\section{2 | DISCUSSION}

We reported the case of a patient with history of BD who developed a progressive supranuclear palsy-parkinsonism (PSP-P) like disease
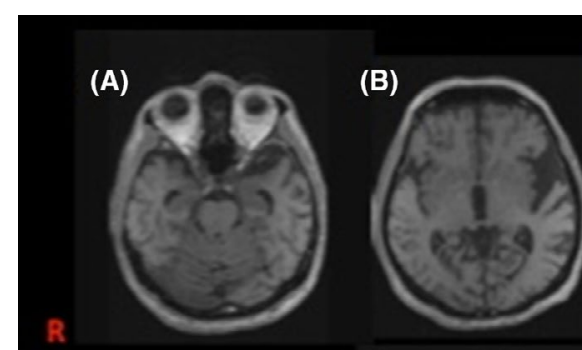

(E)
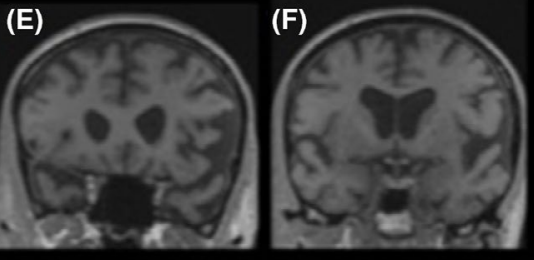

(C)

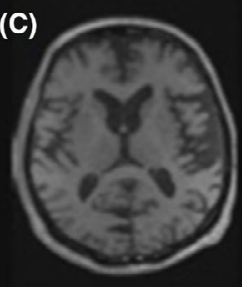

(F)

(G)
(D)

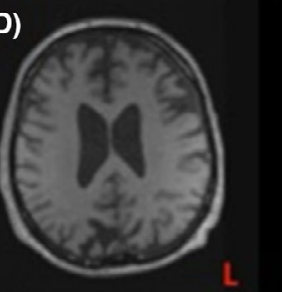

(H)
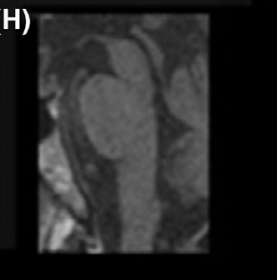
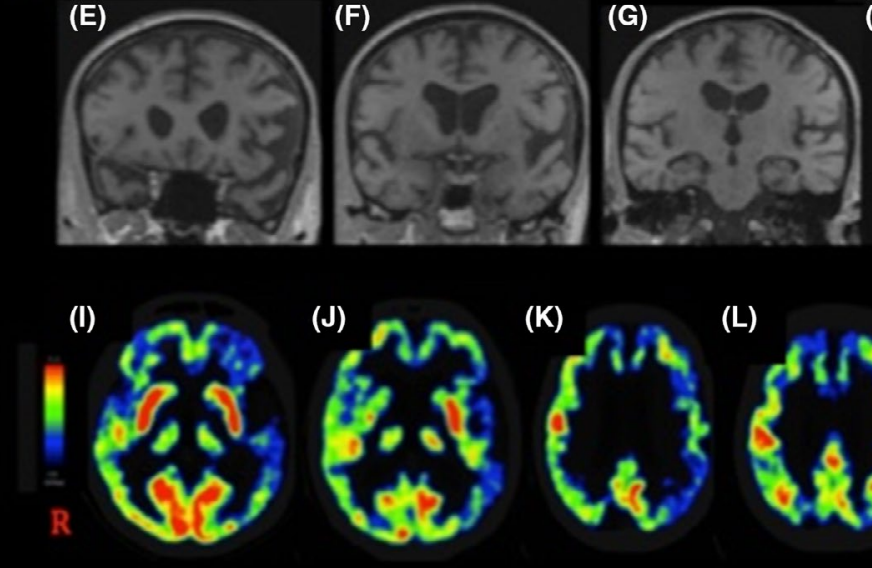

(M)

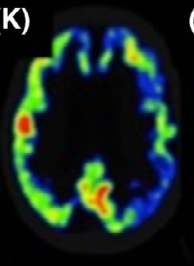

(L)

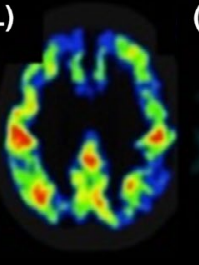

FIGURE 1 T1 weighted magnetic resonance imaging in axial, coronal and sagittal slices. Moderate-to-severe atrophy can be observed in the left lateral temporal regions (B-F), the left insula (F), the left frontal lobe (C-E), especially on the dorsolateral prefrontal cortex (E). Also, a moderate atrophy is present in the mesial bilateral frontal regions on the right (C-E). Additionally, an area on focal atrophy can be appreciated in the right posterior medial region of the parietal lobe (D). The midbrain is mildly atrophic (H). The positron emission tomography with tracer 18-fluorine fluorodeoxyglucose (18-FDG-PET) evidences a diffused hypometabolism in the left frontal dorsolateral area, in the left parietal region and in the left temporal lobe in toto (I-L). The SPECT evaluation shows a decrease in the concentration of I-ioflupane (I-FP-CIT) in both the caudate-putaminal structures, to a greater extent in the left putamen (M) [Colour figure can be viewed at wileyonlinelibrary.com] 
associated with severe brain degeneration, particularly affecting the left frontotemporal lobes (Figure $1 \mathrm{~A}-\mathrm{H}$ ).

The "neuroprogression" of BD has been conceptualized as the progression of neurophysiological and morphostructural alterations in BD patients. However, illness trajectories are largely variable and scarcely predictable. Moreover, no clear neuropathological marker can be ascribable to $\mathrm{BD} .^{1}$ In a recent study, the longitudinal trajectories of frontotemporal dementia (FTD) and psychiatric disorders with behavioural symptoms were examined and the results provide strong evidence of a faster worsening of the symptoms in FTD, with a stable profile in psychiatric conditions. This may provide grounds to assume that our patient's clinical trajectory is due to a frontal lobe neurodegeneration, rather than the evolution of a purely psychiatric condition. $^{2}$ Another recent study, describing the syndromic overlap between frontotemporal lobar degeneration and several psychiatric disorders, reported a similar case. Particularly, a patient diagnosed with BD type I at the age of 33 , was eventually diagnosed with suspected FTD at the age of 68 , presenting with frontal and temporal bilateral atrophy and hypometabolism. ${ }^{3}$

The singular clinical trajectory of the case and the imaging findings rise the question whether the diagnosis of PSP-P is a reliable conclusion or may rather be classified within the spectrum of different neurodegenerative disorders such as frontotemporal dementia (FTD with parkinsonism) or corticobasal degeneration (ie PSP-CBS). Furthermore, the moderate nigrostriatal denervation detected at the DatSCAN could point towards a clinical classification of a PSPmimic rather than a pure PSP.

Cases of BD evolving into FTD have been reported. ${ }^{4}$ However, clinical diagnostic markers for FTD, particularly the behavioural variant (bv-FTD), include early clinical and behavioural symptoms that were lacking at onset and have been absent throughout his illness. Additionally, the genetic analysis was negative for mutation of GRN and C9ORF72, which have been associated with BD-FTD phenotypes. ${ }^{3}$

Although the current criteria for PSP also include forms of PSP with corticobasal phenotype (PSP-CBS), classical cortical signs of CBS were lacking in our patient.
Lithium and neuroleptics drug-induced parkinsonism has been described, but not cases of atypical parkinsonism. Additionally, ocular gaze palsy due to lithium presents with horizontal gaze palsy associated with downbeat nystagmus, instead of vertical gaze palsy.

The width of PSP spectrum is now well-recognized from the clinical and neuroradiological perspective. Furthermore, new genetic mutations in PSP mimics and atypical phenotypes are increasingly acknowledged. ${ }^{5}$

The clinical phenotype expressed by our patient does not allow a reliable diagnosis of PSP or idiopathic PD, therefore, here we propose this case as atypical parkinsonism due to a frontotemporal lobar degeneration developing after a long history of BD.

\section{DATA AVAILABILITY STATEMENT}

The data that support the findings of this study are available from the corresponding author upon reasonable request.

\section{ORCID}

Maria E. Di Battista iD https://orcid.org/0000-0002-6185-9965

Maurizio Gallucci (iD http://orcid.org/0000-0001-6329-3195

\section{REFERENCES}

1. Harrison PJ, Colbourne L, Harrison CH. The neuropathology of bipolar disorder: systematic review and meta-analysis. Mol Psychiatry. 2018. https://doi.org/10.1038/s41380-018-0213-3

2. Reus LM, Vijverberg EG, Tijms BM, et al. Disease trajectories in behavioural variant frontotemporal dementia, primary psychiatric and other neurodegenerative disorders presenting with behavioural change. J Psychiatr Res. 2018;104:183-191.

3. Lanata SC, Miller BL. The behavioural variant frontotemporal dementia (bvFTD) syndrome in psychiatry. J Neurol Neurosurg Psychiatry. 2016;87(5):501-511.

4. Papazacharias A, Lozupone M, Barulli MR, et al. Bipolar disorder and frontotemporal dementia: an intriguing association. J Alzheimers Dis. 2017;55:973-979.

5. Stamelou M, Quinn NP, Bhatia KP. "Atypical" atypical parkinsonism: new genetic conditions presenting with features of progressive supranuclear palsy, corticobasal degeneration, or multiple system atrophy-a diagnostic guide. Mov Disord. 2013;28:1184-1199. 